\title{
Union internationale d'Education pour la Santé
}

\section{Ce qu'elle est}

Fondée en 1951 à Paris, l'Union internationale d'Education pour la Santé est une organisation internationale non gouvernementale qui collabore étroitement avec l'Organisation mondiale de la Santé et l'Unesco, avec lesquelles elle est en relations officielles, ainsi qu'avec l'Unicef et de nombreuses organisations non gouvernementales concernées par la santé.

\section{Ses buts}

L'Union s'efforce de contribuer à l'amélioration de la santé par l'éducation:

1. en servant de lien entre les organisations et les particuliers travaillant dans le domaine de l'éducation pour la santé dans les divers pays et en les aidant à mettre en commun leurs expériences et leurs connaissances;

2. en facilitant les échanges d'information et les confrontations d'expériences à travers le monde pour tout ce qui touche à l'éducation pour la santé, et notamment aux programmes, à la formation professionnelle, à la recherche, aux méthodes et aux techniques, aux moyens de communication, etc.;

3. en favorisant la recherche scientifique et l'amélioration de la formation professionnelle en matière d'éducation pour la santé;

4. en favorisant le développement d'une opinion publique avertie de tout ce qui se rapporte à une existence saine.

\section{Son programme}

Les moyens mis en cuvre par l'Union pour atteindre ces buts comprennent notamment:

- l'organisation périodique de la Conférence internationale d'Education pour la Santé: Paris, 1953; Rome, 1956; Düsseldorf, 1959; Philadelphie, 1962; Madrid, 1965; Buenos Aires, 1969; Paris, 1973; et Ottawa, 1976;

- l'organisation de séminaires régionaux pour l'étude approfondie des moyens d'améliorer la santé par l'éducation;

- la publication trimestrielle de la Revue internationale d'Education pour la Santé en une édition trilingue (français, anglais, et allemand) et d'ouvrages techniques;

- le développement d'activités et de projets dans le cadre de sa Commission de développement technique, qui réunit des techniciens appartenant aux différentes professions dont les recherches et l'action influencent le niveau de santé des populations dans les différentes régions du monde;

- la promotion, par l'intermédiaire de ses membres constituants, collectifs et individuels, d'une participation active des particuliers, des familles et des collectivités à l'amélioration de leur propre bienêtre et de leur santé.

Les membres

L'UIES comprend trois catégories de membres:

Les membres constituants: il s'agit des comités nationaux d'éducation pour la santé ou d'organisations nationales ayant notamment pour vocation de favoriser, de coordonner et de renforcer, dans le secteur qui leur est propre ou sur un plan général, les activités d'éducation pour la santé dans leur pays.

Les membres collectifs: cette catégorie comprend toute collectivité dont les buts et les activités se rapportent à l'éducation pour la santé, notamment des organisations professionnelles travaillant dans le domaine de la santé ou dans des domaines connexes, des institutions exerçant leur activité sur le plan de l'éducation, des fondations, des sociétés industrielles ou commerciales, des associations d'employeurs ou de salariés, etc.

Les membres individuels: il s'agit de personnes ayant un intérêt professionnel ou personnel dans l'éducation pour la santé et qui désirent collaborer activement au développement de tel ou tel aspect du programme de l'Union, ou simplement lui apporter un appui d'ordre général.

Selon la catégorie à laquelle ils appartiennent, les membres de l'Union ont la possibilité:

- de disposer de cinq, trois ou une voix délibérative(s) à l'Assemblée générale;

- de recevoir gratuitement cinq, trois ou un abonnement(s) à la Revue internationale d'Education pour la Santé;

- de recevoir le Bulletin du Secrétariat général;

- d'acquérir à des prix spéciaux les manuels, monographies, etc., publiés par l'Union, et de bénéficier d'un pourcentage sur les commandes en gros;

- de solliciter l'aide du Secrétariat de l'Union en vue d'obtenir des informations sur les programmes, les publications, le matériel audio-visuel d'éducation pour la santé, etc. provenant de différentes régions;

- de confronter leurs expériences dans le cadre des séminaires régionaux et des conférences internationales organisées par l'Union;

- de prendre part à des voyages d'étude à l'étranger, organisés à l'intention de petits groupes de membres de l'Union dans le but de leur donner l'occasion d'étudier des programmes d'éducation pour la santé dans d'autres pays et d'établir de nouveaux liens professionnels tout en goûtant les joies du voyage;

- de patronner conjointement avec l'Union, lorsqu'ils sont membres constituants, des programmes dans le domaine de la recherche ou de la formation professionnelle et des séminaires d'un caractère national ou régional;

- d'inviter, en qualité de comité national, la Conférence internationale d'Education pour la Santé.

\section{Les Cotisations}

Celles-ci varient selon la catégorie de membre et, dans chaque catégorie, selon un barème qui tient compte des aspects économiques. Ainsi, les cotisations des membres collectifs varient de FF 200.- à 625.-, celles des membres individuels de FF 50.- à 125.- et celles des membres constituants sont fixées individuellement. 